International Journal of Engineering \& Technology, 7 (2.31) (2018) 165-169
International Journal of Engineering \& Technology
SPC
Website: www.sciencepubco.com/index.php/IJET
Research paper

\title{
Local energy match based non-sub sampled contourlet Transform for multi modal medical image fusion
}

\author{
M. Shyamala Devi ${ }^{1 *}$, P. Balamurugan ${ }^{2}$ \\ ${ }^{I}$ Associate Professor, Vel Tech Rangarajan Dr. Sagunthala R\&D Institute of Science and Technology, Chennai. \\ ${ }^{2}$ Professor, Vel Tech Rangarajan Dr. Sagunthala R\&D Institute of Science and Technology, Chennai. \\ *Corresponding author E-mail: shyamalapmr@gmail.com
}

\begin{abstract}
Image processing technology requires moreover the full image or the part of image which is to be processed from the user's point of view like the radius of object etc. The main purpose of fusion is to diminish dissimilar error between the fused image and the input images. With respect to the medical diagnosis, the edges and outlines of the concerned objects is more important than extra information. So preserving the edge features of the image is worth for investigating the image fusion. The image with higher contrast contains more edgelike features. Here we propose a new medical image fusion scheme namely Local Energy Match NSCT based on discrete contourlet transformation, which is constructive to give the details of curve edges. It is used to progress the edge information of fused image by dropping the distortion. This transformation lead to crumbling of multimodal image addicted to finer and coarser details and finest details will be decayed into unusual resolution in dissimilar orientation. The input multimodal images namely CT and MRI images are first transformed by Non Sub sampled Contourlet Transformation (NSCT) which decomposes the image into low frequency and high frequency elements. In our system, the Low frequency coefficient of the image is fused by image averaging and Gabor filter bank algorithm. The processed High frequency coefficients of the image are fused by image averaging and gradient based fusion algorithm. Then the fused image is obtained by inverse NSCT with local energy match based coefficients. To evaluate the image fusion accuracy, Peak Signal to Noise Ratio (PSNR), Root Mean Square Error (RMSE) and Correlation Coefficient parameters are used in this work
\end{abstract}

Keywords: Multimodal image, PSNR, RMSE, NSCT, Gabor Filter.

\section{Introduction}

Medical image fusion is the process in which two input images with incomplete information are transformed into a single fused output image with the high resolution and complete information. In the medical images, CT scan can evidently mirror the anatomical organization of bone tissues. Oppositely, MRI can clearly reflect the anatomical arrangement of soft tissues, organs and blood vessels. CT, MRI and other modes of medical images reflect the human information from various angles. In the clinical diagnosis and treatment, the problems about the comparison and synthesis between image CT and MRI were frequently encountered. The actual fusion process is done at different levels of information representations like signal, pixel, feature and symbolic level. The pixel level image fusion is measured principally to be existing to the human observer, especially in image sequence fusion.

\section{Fusion methods}

In the field of medical imaging, we are in need to process various scan images of the identical part of the same patient with different scan imaging equipments. And also, the information given by diversity of imaging method is often matching. In the medical images, CT can clearly replicate the anatomical structure of bone tissues. Whereas the MRI can obviously imitate the anatomical construction of soft tissues, organs and blood vessels. CT, MRI and other modes of medical images reveal the human information from various angles. So in the clinical diagnosis and treatment, we need to compare and perform synthesis between image CT and MRI images. This leads to the practise of image fusion methods.

\subsection{Linear superposition}

In this method fused image is developed from various input frames as a subjective superposition of each input frames. The optimized weighting coefficients with deference to information content and duplication exclusion are done by principal component analysis (PCA) of all input intensities. By executing a PCA of the covariance matrix of input intensities, the weightings for each input frame are obtained from the eigen vector equivalent to the biggest eigen value.

\subsection{Non Linear methods}

This method creates the complex fused image by using the features of the simple nonlinear operator such as max or min. The same approach can be applied with image algebra. The image algebra concept is a high-level algebraic extension of image morphology that is considered to portray all image processing operations.

The basic variety defined in image algebra is value sets, coordinate sets that allows the amalgamation of dissimilar resolutions, images and templates. 


\subsection{Optimization methods}

Here the image fusion is expressed as an bayesian optimization problem. The main purpose is to find the fused image which maximizes the posterior probability. In this method, all the input images are modeled as markov casual fields with the comparison of gibbs random fields and markov random fields. This is the energy function can be articulated as clique potentials. In Clique potentials, the pixels affect the actual pixel value. The maximization of the energy function is then done in the fused image.

\subsection{ANN methods}

The researchers also use artificial neural networks in the procedure of pixel-level image fusion. The trendy pattern for image fusion of dissimilar imaging sensors in organic systems is described by Newman and Hart line in the 80s: Rattlesnakes acquire a method namely pit organs which are responsive to thermal radiation through a dense network of nerve fibers.

\subsection{Image pyramid}

Image pyramids have been initially portrayed for multi resolution image analysis and as a model for the binocular fusion in human vision. Image pyramid is a series of images where each image is constructed by low pass filtering and sub sampling from its predecessor.

Due to sampling, the image size is bisected in both spatial directions at each level of the decomposition process, thereby resulting denoting the multi resolution signal representation.

\subsection{Drawbacks of the existing methods}

- Contrast information loss due to averaging method.

- Maximizing approach is sensitive to sensor noise and high spatial distortion.

- The adoption of $\mathrm{K}$ means algorithm is not applicable for all lighting condition of images.

- Difficult to measure the cluster quality.

\section{Local energy match NSCT}

\subsection{Proposed system architecture}

In our Local Energy Match NSCT System, the input images are processed by Non Sub sampled Contourlet Transformation (NSCT) to form low frequency and High Frequency components. Then the low frequency elements of both the images are processed by the Gabor filter bank algorithm to find the gabor value. The processed low frequency elements are picked based on the maximum gabor value. Similarly the high frequency elements of both the images are processed by Gradient algorithm to find the gradient value. The processed high frequency elements are picked based on the maximum gradient value. The System architecture of our system is shown in Fig 2. The advantages of our proposed system are shown below.

- The memory cost is low.

- It improves the detection of edges and texture region than other transforms.

- Effective performance for low contrast image.

- High performance result for overlapped data.

\subsection{Modules of local energy match NSCT}

In our Local Energy Match NSCT System, the functional modules are as follows,
a) NSCT Module.
b) Gabor Filter Module.
c) Gradient Filter Module.

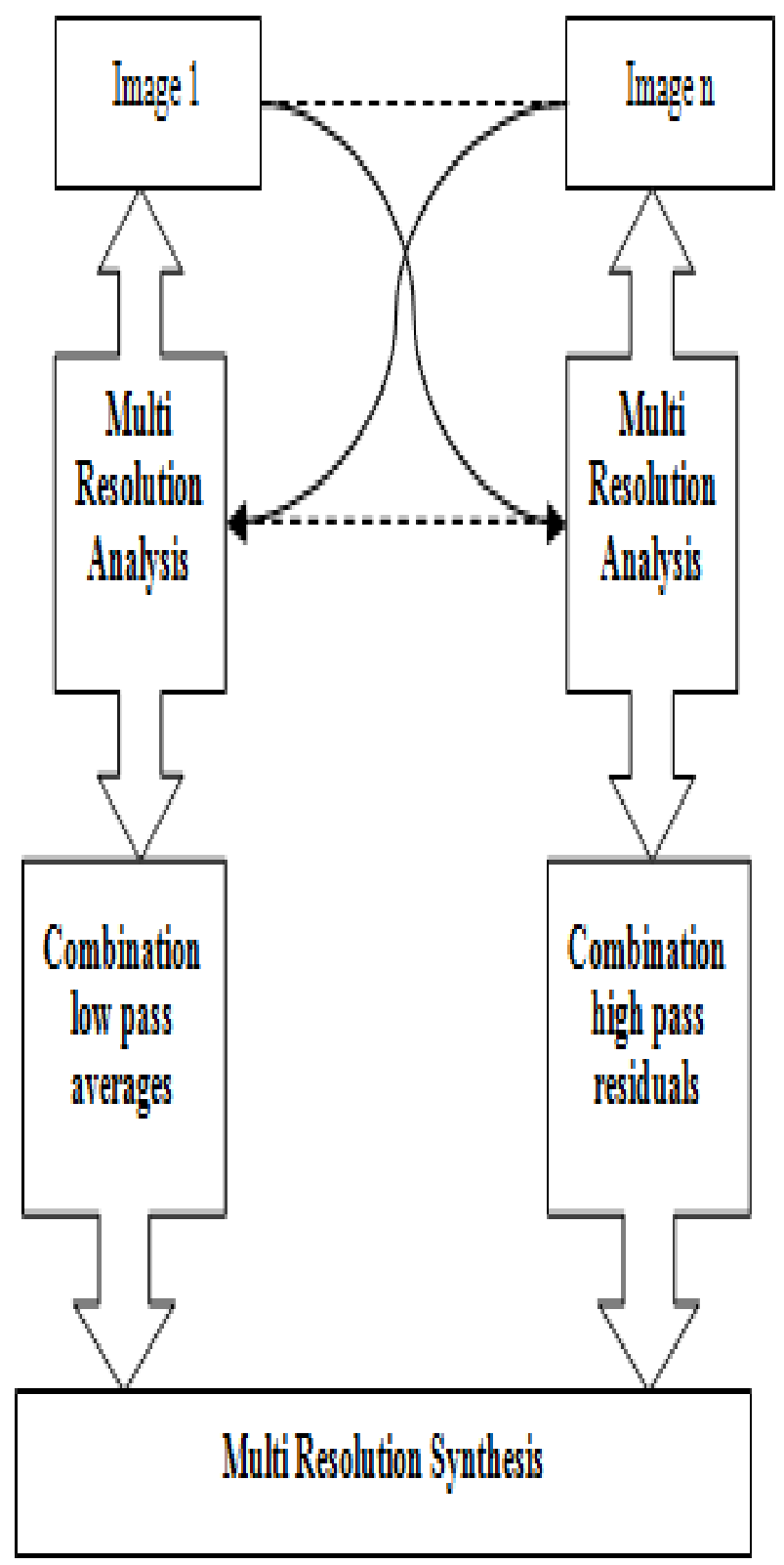

Fig. 1: Block diagram of image fusion

\subsubsection{NSCT module}

Algorithm 1: NSCT Filter

[1] Get the input image 1,2

[2] Obtain the low frequency components and the high frequency components by using fourier transform

[3] Form the low frequency and high frequency subband Coefficient of image 1,2

[4] Find the standard deviation of low frequency and high frequency subband co-efficient.

[5] Obtain edge values by edge detection of image 1,2 .

[6] Perform dual threshold segmentation by fuzzy C Means algorithm for low frequency and high frequency image components

[7] Then apply Fusion rules to complete the partition of low frequency and high frequency elements 


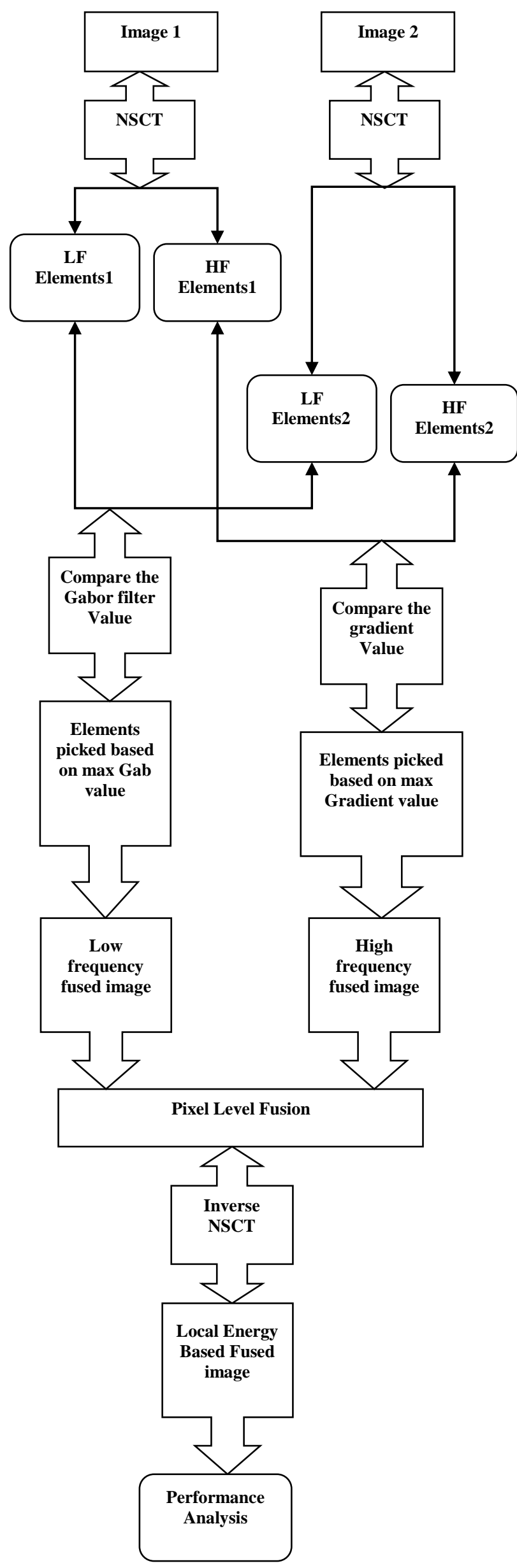

Fig. 2: System architecture of local energy match nsct system

\subsubsection{Gabor Filter Module}

Algorithm 2: Gabor Filter

[1] Get the Low Frequency element of image 1, 2

[2] Divide the LF of image 1 into $16 \times 16$ blocks

[3] Divide the LF of image 2 into $16 \times 16$ blocks

[4] Compare the features of 4 different scales at 8 various angles of LF image 1,2

[5] Obtain the 8 different angles of LF image 1,2

[6] Claculate mean and Standard deviation to find the Gabor value.

\subsubsection{Gradient Filter Module}

Algorithm 3: Gradient Filter

[1] Get the High Frequency element of image 1, 2.

[2] Divide the HF of image 1 into $16 \times 16$ blocks with respect to $\mathrm{X}$ direction.

[3] Divide the HF of image 2 into $16 \times 16$ blocks with respect to Y direction.

[4] Compute the fist order derivative of HF image with respect to $\mathrm{X}$ direction.

[5] Compute the fist order derivative of HF image with respect to Y direction.

[6] Compute the gradient value using sobel operator.

[7] Pick the High frequency elements based on maximum gradient value.

\section{Results and discussion}

\subsection{Implementation}

Our system Local Energy Match NSCT System is implanted with MATLAB and the performance evacuation is done for the following metrics.

1. Peak Signal to Noise Ratio.

2. Root Mean Square Error.

3. Correlation Coefficient.

\subsubsection{Peak Signal to Noise Ratio}

Peak Signal to Noise Ratio is defined as the ratio between the maximum power of the signal and the fidelity noise of the image. The PSNR is calculated to analyse the similarity of the images.

$$
\mathrm{PSNR}=10 * \log 10(255 * 255 / \mathrm{MSE})
$$

Where

MSE = Mean Square Error

Mean Square Error is the difference between the fused image and the original image.

\subsubsection{Root Mean Square Error}

Root Mean Square Error is defined as the inference between the fused image and the original image based on the percentage of mean intensity of the original image.

$$
R M S E=\frac{1}{M N} \sum_{i=0}^{M-1} \sum_{j=0}^{N-1}[O I(i, j)-F I(i, j]
$$

Where

$\mathrm{OI}(\mathrm{i}, \mathrm{j})=$ Pixel Values of Original image at position $\mathrm{i}, \mathrm{j}$ 
FI $(i, j)=$ Pixel Values of Fusedl image at position $i, j$

$\mathrm{M}=$ Number of rows in the image

$\mathrm{N}=$ Number of columns in the image

\subsubsection{Correlation Coefficient}

Correlation Coefficient is done to find the similarity of original and fused image and it is done by the following

Correlation $=\frac{\operatorname{Sum}((F I-\text { Mean of } F I) *(O I-\text { Mean of OI }))}{\operatorname{Sqrt}(((F I-\text { Mean of FI }) *(O I-\text { Mean of OI })))}$

The Results are shown below.
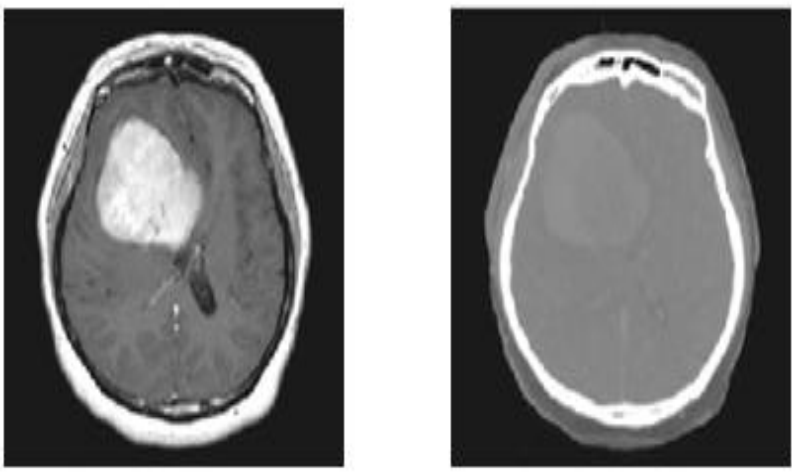

Fig. 3: Input image (CT and MRI Image)

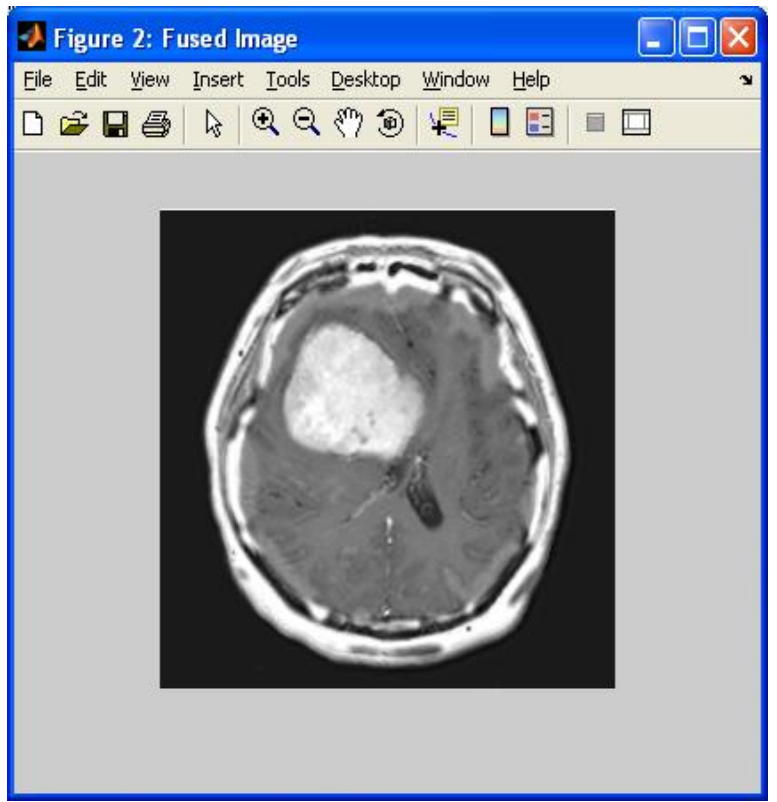

Fig. 4: Fused Image based on local energy match NSCT

Table 1: Performance Analysis of Local Energy Match NSCT System with Existing Fusion methods

\begin{tabular}{|l|c|c|c|}
\hline \multirow{2}{*}{ Fusion Methods } & \multicolumn{3}{|c|}{$\begin{array}{c}\text { Input Image } \\
\text { (CT and MRI Image) }\end{array}$} \\
\cline { 2 - 4 } & RMSE & PSNR & $\begin{array}{l}\text { Correlation } \\
\text { Coefficient }\end{array}$ \\
\hline $\begin{array}{l}\text { Ripplet Transform Method } \\
\text { (RT) }\end{array}$ & 5.2 & 31.2 & 0.52 \\
\hline $\begin{array}{l}\text { Wavelet Transform Meth- } \\
\text { od (WT) }\end{array}$ & 4.9 & 33.2 & 0.56 \\
\hline $\begin{array}{l}\text { Curvelet Fusion Method } \\
\text { (CF) }\end{array}$ & 4.3 & 35.4 & 0.62 \\
\hline $\begin{array}{l}\text { NSCT with DCT } \\
\text { Local Energy Match } \\
\text { NSCT Method (LEMN) }\end{array}$ & 3.5 & 42.8 & 0.85 \\
\hline
\end{tabular}

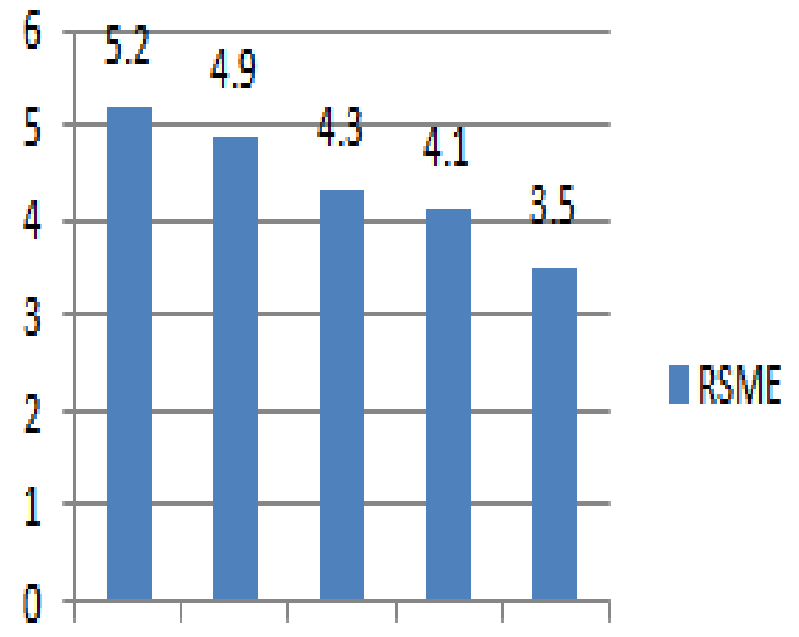

RT WT CF NSCT LEMN

Fig. 5: RSME analysis of local energy match NSCT

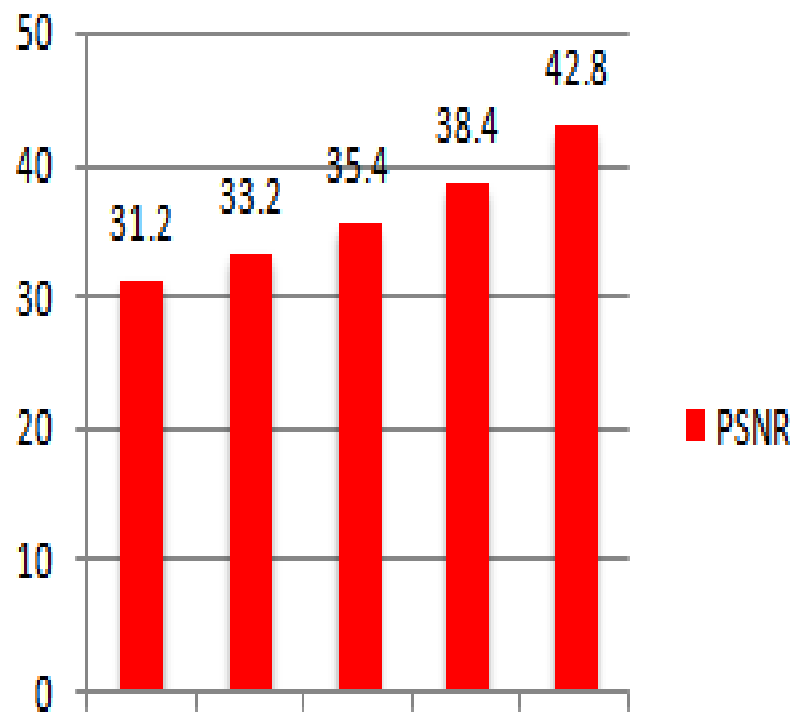

RT WT CF NSCT LEMN

Fig. 6: PSNR analysis of local energy match NSCT Correlation

Coefficient

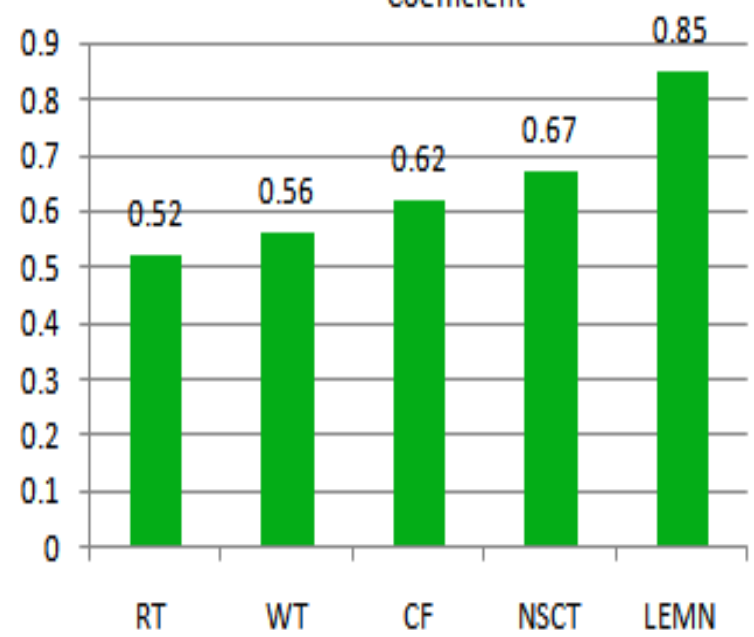

Fig. 7: Correlation analysis of local energy match NSCT 


\section{Conclusion}

In this paper, an image fusion method Local Energy Match NSCT is proposed. The input images are processed by NSCT to form low frequency and High Frequency components. Then the low frequency elements of both the images are processed by the Gabor filter bank algorithm to find the gabor value. The processed low frequency elements are picked based on the maximum gabor value. Similarly the high frequency elements of both the images are processed by Gradient algorithm to find the gradient value. The processed high frequency elements are picked based on the maximum gradient value. Our method is also evaluated with the qualitative measure of PSNR, RSME and Coorelation coefficient. The future work is to maximize the PSNR by developing wavelet method.

\section{References}

[1] Petrovic VS \& Xydeas CS, "Gradient-based multiresolution image fusion", IEEE Trans. Image Process., Vol.13, no.2, (2004), pp.228-237, Feb.

[2] Li H, Manjunath BS \& Mitra SK, "Multi sensor image fusion using the wavelet transform", Graph Models Image Process., Vol.57, No.3, (1995), pp.235-245.

[3] Bhatnagar G \& Raman B, "A new image fusion technique based on directive contrast", Electron. Lett. Comput. Vision Image Anal., Vol. 8, No.2, (2009), pp.18-38.

[4] Zhang Q \& Guo BL, "Multi focus image fusion using the non subsampled Contourlet transform", Signal Process., Vol.89, No.7, (2009), pp.1334-1346.

[5] Ardizzone E, Pirrone R, \& Orazio OG, "Fuzzy C-Means Segmentation on Brain MR Slices Corrupted by RF-Inhomogeneity", Proc. The 7th international workshop on Fuzzy Logic and Applications: Applications of Fuzzy Sets Theory, (2007), pp.378-384

[6] Bianrgi PM, Ashtiyani M \& Asadi S, "MRI Segmentation Using Fuzzy C-means Clustering Algorithm Basis Neural Network", In Proc. ICTT A $3^{\text {rd }}$ International Conference on Information and Communication Technologies: From Theory to Applications, (2008), pp.1-5. 